\title{
Computationally Grafting an IgE Epitope onto a Scaffold: Implications for a Pan Anti-Allergy Vaccine Design
}

\author{
Sari Sabban ${ }^{1 *}$
}

\begin{abstract}
Allergy is becoming an intensifying disease among the world population, particularly in the developed world. Once allergy develops, sufferers are permanently trapped in a hyper-immune response that makes them sensitive to innocuous substances, such as the protein cupin in peanuts. The immune pathway concerned with developing allergy is the $\mathrm{Th}_{2}$ immune pathway where the IgE antibody (targeting innocuous substances) binds to its FceRI receptor on Mast and Basophil cells. Currently, there is not a permanent treatment for allergies. This paper discusses a strategy and a protocol that could disrupt the binding between the antibody and its receptor for a potential permanent treatment. Ten proteins were computationally designed to display a human lgE motif very close in proximity to the $\mathrm{IgE}$ antibodies's FceRI receptor's binding site in an effort for these proteins to be used as a vaccine against our own IgE antibody. The motif of interest was the FG motif and it was excised and grafted onto a Staphylococcus aureus protein (PDB ID 1YN3). The new structures (motif + scaffold) had their sequences re-designed around the motif to find an amino acid sequence that would fold to the designed structures correctly. These ten computationally designed proteins showed successful folding when simulated using Rosetta's AbinitioRelax folding simulation and the lgE epitope was clearly displayed in its native threedimensional structure in all of them. These designed proteins have the potential to be used as a pan anti-allergy vaccine by guiding the immune system towards developing antibodies against the body's own IgE antibody, thus neutralising it, and presumably permanently shutting down a major aspect of the $\mathrm{Th}_{2}$ immune pathway. This work employed in silico based methods for designing the proteins and did not include any experimental verifications.
\end{abstract}

\section{Keywords}

Protein Design — Epitope Grafting — Vaccine Design — Computational Structural Biology — Allergy — Type I Hypersensitivity

${ }^{1}$ Department of Biological Sciences, Faculty of Science, King Abdulaziz University, Jeddah, Makka, Kingdom of Saudi Arabia *Corresponding author: sari.sabban@gmail.com

\section{Introduction}

Allergy was first defined by Clemens von Pirquet in 1906 when he discovered that second injections of horse serum caused a severe inflammatory reaction in some, but not all, individuals. He termed this condition Allergy, from the Greek words allos "other" and ergon "works" and therefore the allergy causing agent was called an "allergen" [7]. In the 1960s Kimishige Ishizaka and Teruko Ishizaka demonstrated that allergic reactions are mediated by a new class of antibodies that they discovered and called immunoglobulin E [18] [12], which binds onto a receptor called the high-affinity $\operatorname{IgE}$ receptor (FceRI) which is found on Mast and Basophil cells and comprises four chains (an $\alpha$ extracellular chain with two domains, an intermembrane $\beta$ chain, and two intermembrane $\gamma$ chains protruding into the cytoplasm).

Humans have five antibody types (IgA, IgD, IgE, IgG, and $\operatorname{IgM})$. immunoglobulin $\mathrm{G}(\operatorname{IgG})$ is the most abundant antibody type since it targets viral and bacterial pathogens.
Immunoglobulin E (IgE) on the other hand, is concerned with parasitic immunity. Since parasites are eukaryotes and closer to other eukaryotes phylogenetically compared to bacteria and viruses, this pathway can target innocuous substances that look like parasites but are not usually harmful, leading to a type of inflammatory reaction termed an allergic reaction, or known medically as type I hypersensitivity.

Thus, IgE antibodies are best known for their role as mediators of the allergic response, which in its most serious manifestations, causes asthma or an anaphylactic shock. Reports of an increase in the number of individuals suffering from allergic manifestations began in the second half of the last century and the incidence of allergy has now reached pandemic proportions [21]. IgE mediated allergic responses have diverse manifestations, which range from mild to severe and can be life threatening. Mammals including humans, dogs, and horses are known to suffer the clinical symptoms of IgE-mediated type I hypersensitivity responses. In spite of extensive worldwide research efforts, no effective active 
therapeutic intervention strategies are currently available.

One of the perceived reasons for the continual increase in allergy incidence, especially in the developed world, is a hypothesis termed the Hygiene Hypothesis, originally formulated by Strachan [26] [27] [20], it states that a lack of exposure to infectious pathogens in early childhood, i.e. living in an environment too clean, can lead to inadequate immune system development, i.e. a shift from the $\mathrm{Th}_{1}$ immune response (bacteria, viruses) to that of the $\mathrm{Th}_{2}$ immune response (parasite, allergy), increasing the susceptibility to develop allergy. Further studies in this immunological pathway has shed light into the viability of this hypothesis and showed a correlation between tuberculosis infections in childhood and lack of allergy in adulthood [28].

Currently, the most widely used therapy against allergy is pharmacotherapy, which is a passive immunotherapeutic intervention strategy employing the use of anti-histamines, corticosteroids, or epinephrine, all of which alleviate the symptoms of allergy without curing its underlying cause. The quest to treat allergy is not a new concept, it was first attempted in 1911 [14] when subcutaneous injections of an allergen extract were administered in an effort to desensitise atopic patients to certain allergens. This procedure was successful to treat certain conditions such as anaphylaxis and allergic rhinitis, but it was unsuccessful in treating asthma [17]. This protocol has remained controversial as it has the potential to sensitise patients even more, thus worsening their condition [19]. A novel immunotherapy called sublingual immunotherapy is currently being researched where allergen extracts are given to patients under their tongues [10]. The efficacy of these therapies varies greatly between individuals since doctors do not have a standardised protocol to follow, they usually develop their own protocols according to their own observations and individual successes.

Since allergy incidence have been on the rise globally, a new form of therapy is under development. Though still a passive immunotherapeutic strategy, it employs non-anaphylactogen antibodies which have demonstrated their capacity to treat type I hypersensitivity responses. These humanised mouse monoclonal antibodies (mAbs), of which Omalizumab [22] is best characterised, are now successful in treating severe forms of allergy, but have been shown to be associated with a number of drawbacks: 1) poor effectiveness in obese patients, 2) logistics and cost, 3) treatment only reduces symptoms temporarily, hence it is a passive immunotherapeutic strategy.

These draw backs logically lead to the potential to develop new active forms of immunotherapeutic strategies, such as a vaccine that primes the immune system against its own $\operatorname{IgE}$ antibody, at which point the $\operatorname{IgE}$ is neutralised and the allergy disease is terminated. Even though current mainstream research is concentrating on the passive immunisation approach, it is believed that active immunisation is a viable form of treatment against this disease (figure 1).

This paper proposes a strategy by which a pan-anti allergy vaccine can be computationally designed by excising the mo- tif of interest from the IgE structure and grafting in onto a scaffold protein structure, thus displaying only the motif of interest in its original three dimensional form without any of the surrounding native structure, allowing the immune system to target just that particular motif.

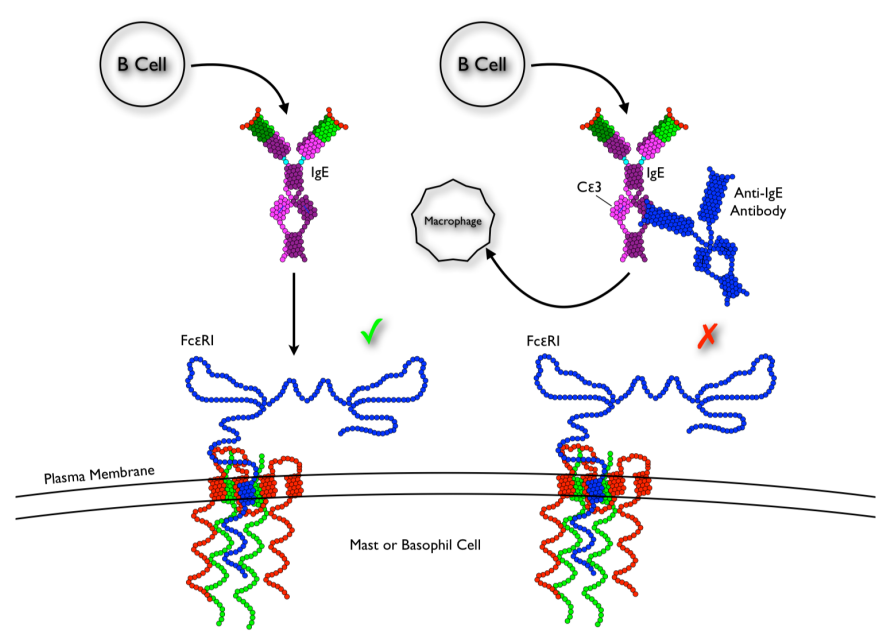

Figure 1. Summery of the pan-anti allergy vaccine therapy concept. Administering a vaccine that is capable of producing antibodies against the body's own self IgE molecule, and neutralising it by preventing it from binding onto its receptor, could disrupt the entire allergy pathway and potentially curing the disease [30].

\section{Methods}

The following steps were used to generate a database of scaffold protein structures to search for a an appropriate backbone to graft the motif onto, as well as isolate the IgE motif, graft it onto the found scaffold, then design the scaffold to fold onto the designed protein structure.

\section{Motif determination and excision}

The FG motif (figure 2 purple colour) from the human $\operatorname{IgE}$ crystal structure (PDB ID 2Y7Q, chain B, amino acids 420429 with the sequence VTHPHLPRAL) was chosen due to its very close proximity to the IgE's receptor binding site (PDB ID 2 Y7Q, chain B, amino acids 331-338 with the sequence SNPRGVSA) named the R loop (figure 2 green colour). The FG motif has a ridged structure and it forms a beta sheet loop with an anchoring lysine at position 425 pointing into the core fixing its shape. Since the IgE's receptor binding site (R loop) was not anchored in place with an amino acid pointing into the core it had a higher degree of movement and thus was not well modelled in the crystal structure, hence it was not chosen (this can be clearly observed when looking at chain $\mathrm{C}$ where the same position is missing), furthermore, when the $\mathrm{R}$ loop was grafted it assumed multiple structures as can be seen from 2, thus the FG loop was chosen instead. The FG motif was isolated along with the full receptor chain (PDB ID 2Y7Q all of chain A) as separate .pdb files in preparation for grafting. In the 2Y7Q crystal structure only the receptor's extracellular $\alpha$ chain is modeled with its two domains. 


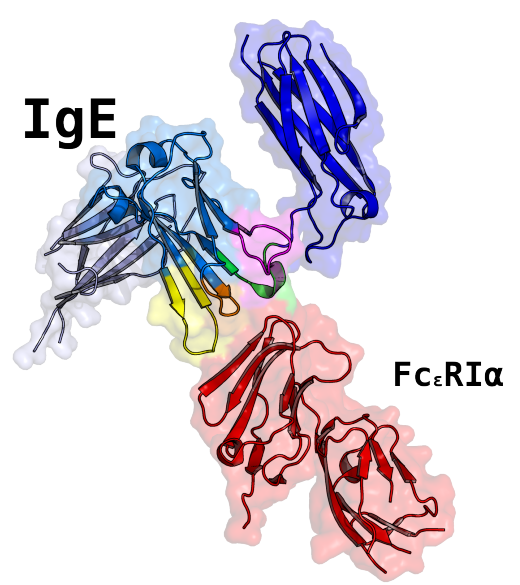

Figure 2. The structure of the human IgE bound to its Fc $\varepsilon$ RI $\alpha$ receptor (2Y7Q). The colours show the different loops that are closet in proximity or forms hydrogen bonds with the receptor when bound. Purple for the FG loop, green for the R loop, orange for the BC loop, and yellow for the DE loop.

\section{Scaffold database generation}

The scaffold database was generated by downloading the entire protein databank using this command:

rsync - rlpt $-\mathrm{v}-\mathrm{z}$-delete - port $=33444$ rsync. wwpdb. org : : ftp data/structures/divided/pdb/ ./PDBdatabase

Each structure was unzipped and the original zipped structured deleted to save memory space. Then, each structure with multiple chains was separated into separate .pdb files using this simple python script that uses the biopython [32] python library:

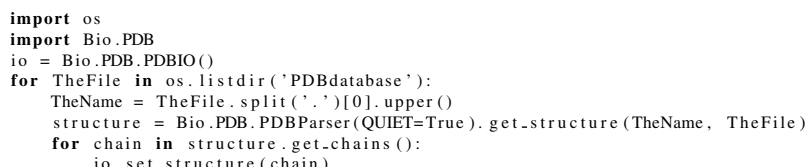

Then desired structures (sizes below 150 amino acids) were isolated using the following bash code:

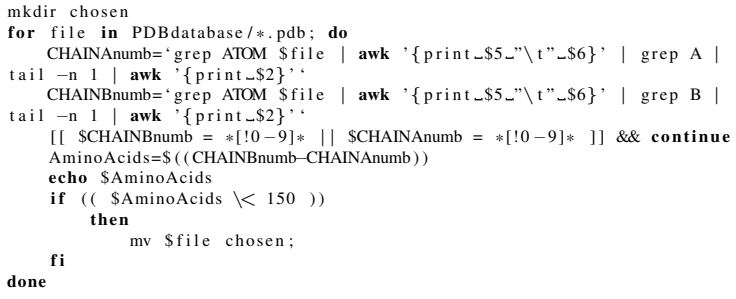

Then, structures were cleaned (removed of any nonepeptide atoms) using the following Linux terminal bash command:

grep -e ATOM - e MSE -F PDBID_CHAIN. pdb > PDBID_CHAIN_clean.pdb

for example:

grep -e ATOM -e MSE -F 3HZ7_A.pdb > 3HZ7_A_clean.pdb

Care must be taken to ensure that the non-canonical MSE (selenomethionine, which is used to solve crystal structures) amino acid is transferred to the cleaned structure which is under the HETATM heading and the MSE amino acid code in .pdb file instead of ATOM heading and the MET amino acid code for methionine. If MSE was not imported it will result in structures with missing selenomethionine. To ensure that the structures are compatible with PyRosetta and will not crash it, they are run through this script (basically just imported then exported):

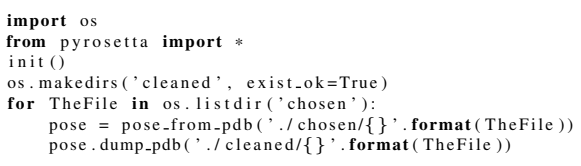

Structures that were not satisfactory were deleted. In this way the scaffold database was constructed.

\section{Scaffold search and motif grafting}

The desired IgE motif (the FG motif) with the sequence VTHPHLPRAL between positions 420 and 429 in chain B of the protein crystal structure with PDB ID 2Y7Q was isolated along with all of chain A, which was the FceRI receptor's $\alpha$ chain since in the 2 Y7Q crystal structure only the receptor's extracellular $\alpha$ chain was modeled with its two domains. A scaffold search was performed where the motif was grafted onto each structure within the scaffold database using the epitope grafting protocol [3] [2]. If there was a match within an RMSD value of $1.0 \AA$ or less the grafted structure (with the motif replacing the original backbone on the scaffold) was measured for its clash with the receptor (i.e: to make sure the backbone was not grafted inward or was buried within the structure). If there was no clash with the receptor structure, then the final grafted structure was exported. The code used for this step can be found in this GitHub repository.

\section{Selective fixed-backbone sequence design}

The final grafted structure was tested for folding (in the next section), which failed to converge into a low root-mean-square deviation (RMSD) low free energy score. Thus, to find a sequence that would allow the grafted structure to fold into the desired structure it had to be sequence designed, i.e: find a sequence that would fold into the desired structure. Initially, this was attempted manually by human guided mutations where amino acids were mutated at a strategic location chosen visually to fill in core voids using only amino acids that were specific to the secondary structures of the mutation sites and their layer position calculated by each amino acid's surface accessible surface area (SASA) using the same criteria in [33]. After several failed attempts, the RosettaDesign fixedbackbone design protocol was employed [13] [8] [1] [11] [15]. The side chains (amino acid identities) of the structure were stochastically mutated and packed using a rotamer library to find the lowest energy structure that would fold into the designed structure. In this protocol, the REF2015 energy function weights were changed to include aa_rep 1.0, aspartimid_penalty 1.0, buried_unsatisfied_penalty 1.0 , and approximate_buried_unsat_penalt 5.0, which assisted in designing an adequate sequence that fits the backbone structure 
and increased the energy gap between the desired structure and any other possible undesired fold. The code used for this step can be found in this GitHub repository.

\section{Folding simulation}

To get insight into whether or not the design process was successful, the folding of the sequence-designed-grafted-structure was simulated using the Rosetta [16] AbinitioRelax protocol [23] [6] [4] [5] [24] [25], which employs a Monte Carlo method, where the amino acid sequence is used to construct a straight primary structure, then 3-mer and 9-mer fragments are randomly inserted, the fragments were generated from the Robetta fragment server (http://robetta.bakerlab.org/fragmentsubm using the amino acid sequence. These fragments are backbone torsion angles of secondary structures that were statistically calculated from the amino acid sequence and they help speed up the simulation. Then the structure is randomly moved (backbone and side chain torion angles changed) and its free energy calculated using the REF2015 scoring function which employs first physical principles and some statistical weights [31] using the following equation (details are explained in the original paper):

$$
\Delta E_{\text {total }}=\sum_{i} w_{i} E_{i}\left(\Theta_{i}, a a_{i}\right)
$$

After several cycles of moving and scoring the final structure was exported. This was repeated 1 million times, which results in 1 million simulated structures. These structures are then plotted on a score vs RMSD plot to show how similar they are to the original designed structure. A successful simulation would result in a funnel-shaped plot, where the lowest scoring structures (lowest free calculated energy) results in structures close to the designed structure (low RMSD) since it is assumed that any protein structure resides in the global free energy minima. The code used for this step can be found in this GitHub repository.

\section{Results}

Analysis of several motif positions revealed that the $\mathrm{R}$ loop and the FG loop from the human IgE (PDB ID 2Y7Q) are the best candidates for a targeted vaccine due to their proximity to the binding site on the $\alpha$ chain of the FceRI receptor (figure 2). After several attempts at grafting and designing the $\mathrm{R}$ loop onto a Mycobacterium smegmatis EsxGH protein (PDB ID 3Q4H replacing the sequence QGDTGMTY at positions 44-51), the FG loop appeared to be the better choice, this was due to the FG loop having an inward pointing leucine, resulting in a ridged loop structure, compared with the $\mathrm{R}$ loop that had a high degree of angle freedom, which resulted in a wide range of different structures when grafted, see figure 3 for a comparison between the grafted and designed motif coloured in purple.

The scaffold search resulted in the FG loop motif being grafted onto a Staphylococcus aureus EAP protein (PDB ID
1YN3) [9] structure as well as several other structures (figure 4). The 1YN3 structure was chosen because it had a backbone that was easily simulated by forward folding using the AbinitioRelax protocol (figure 5) when tested as a control on the original wild type crystal structure. Another reason was that the $1 \mathrm{YN} 3$ protein is a Staphylococcus aureus protein which was expressed in Escherichia coli when it was crystallised and is highly antigenic, thus it is predicted to easily crystallise for final structural evaluation and the backbone could instill a strong immune response, which is required to develop antibodies that would bind to the $\operatorname{IgE}$ at a stronger affinity than the IgE binds onto its receptor. The motif was it.jgpafted between positions 164 and 173 on the 1YN3 structure replacing the sequence ITVNGTSQNI with VTHPHLPRAL (figure 6). As predicted the freshly grafted structure failed a forward fold simulation using AbinitioRelax, this was due to the addition of the motif backbone and side chains severely disrupted the stability of the entire structure. To overcome this, the entire structure was sequence designed by changing and optimising the side chains (except for the motif) while fixing the backbone to stabilise the structure and accommodate the new motif backbone and side chains. At first, manual sequence design was performed, which proved fatal, then the RosettaDesign protocol was successfully used as described in the methods section. Since a failure rate exists between a successful forward fold and a successful crystal structure, the sequence design step was repeated ten times, this resulted in ten structures with the same motif and backbone but different sequences, all of which had a successful forward folding simulation (figure 7). This should increase the probability of synthesising a correctly folded vaccine structure since only one of these structures must pass a crystallography evaluation to be tested as a potential vaccine. If several structures do pass the crystallography evaluation, one structure can be used as a vaccine, while the others are used as a boosts.

The following are the sequences of all the structures, aligned with each other to highlight the differences:

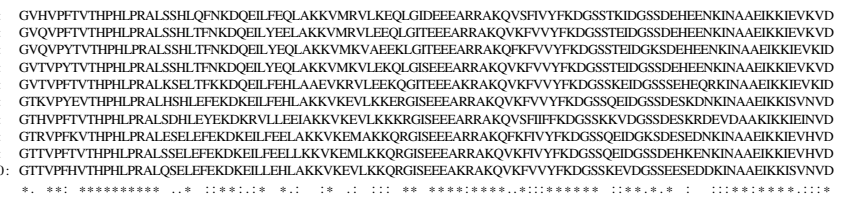

To analyse the structures further, all their FASTA sequences were used to predict the secondary structures of the final proteins. The following are the predicted secondary structures using PSIPRED [34] (H for helix, E for Strand, and C for Coil), des is for the designed structure's secondary structures and pre is for predicted secondary structures from the designed structure's amino acid FASTA sequence:

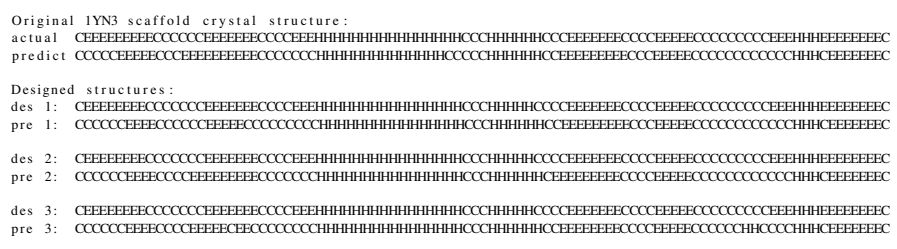


bioRxiv preprint doi: https://doi.org/10.1101/2020.09.14.296392; this version posted March 11, 2021. The copyright holder for this preprint (which was not certified by peer review) is the author/funder. All rights reserved. No reuse allowed without permission.

Computationally Grafting an IgE Epitope onto a Scaffold: Implications for a Pan Anti-Allergy Vaccine Design — 5/9

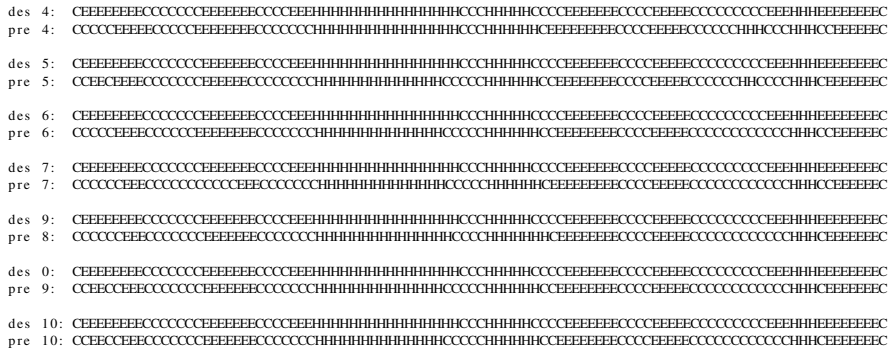

Furthermore, the SWISS-MODEL tool was use to predict the structure of the designed structure from their FASTA sequence as a way to further evaluate their structures computationally. All proteins were predicted to fold as their designed structures 8 .

All structures were predicted to fold within a sub angstrom level of the designed structure, giving high confidence that these will be the structures of the proteins when physically synthesised. Each structure must be crystallised and confirmed the correct fold of the protein and the motif before they can be tested on animals.

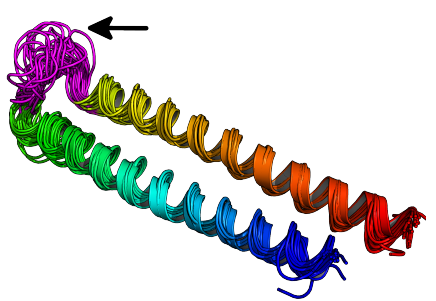

(a)

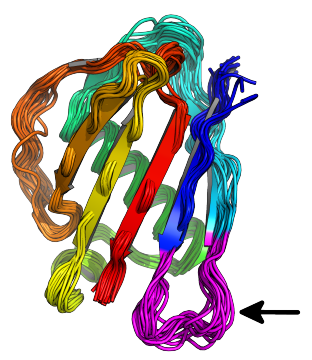

(b)
Figure 3. Comparison of grafting the $\mathbf{R}$ motif to grafting the FG motif. A: Folding simulation of the $\mathrm{R}$ loop motif (in purple) after successfully being grafted onto the $3 \mathrm{Q} 4 \mathrm{H}$ protein and sequence designed, here showing a large variability of the motif backbone since it lacked an anchor (average RMSD = $1.29 \AA$ to the natives motif). B: Folding simulation of the FG loop motif (in purple) after successfully being grafted onto the $1 \mathrm{YN} 3$ protein and sequence designed, here showing better motif stability (average RMSD $=0.62 \AA$ to the natives motif). Structures rendered through PyMOL [29]

Molecular Dynamics simulations [36] [37] [38] [39] [40] [41] [42] [43] were performed on all structures to test the stability of the folded designed structures. Initially the simulation was performed at $300^{\circ} \mathrm{K}$ using a 0.002 femtosecond time step for 100 nanoseconds (figure 9), all the structures showed an RMSD value around $2 \AA$. Structures 3, 4, 5, 6, and 8 showed the highest stability (RMSD values mostly less than $2 \AA$ ) and a radius of gyration value less than $13 \AA$. while structures 7, 9, and 10 showed the lowest stability (RMSD values reaching above $2 \AA$ but less than $3 \AA$ at the end of the simulation). The radius of gyration for all structures was around $13 \AA$. Then the simulation was performed at $400^{\circ} \mathrm{K}$ using the same parameters to test if the structures would unfold (figure 10). The structures showed less RMSD stability (fluctuating up to $4 \AA$ and $5 \AA$ ). Structures $1,2,3,4,6,7,8,9$, and 10 showed low stability by reaching higher RMSD values than the $300^{\circ} \mathrm{K}$ simulation, while structure 5 showed the highest

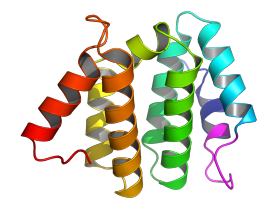

(a)

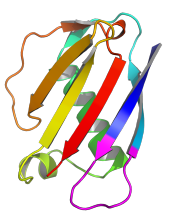

(b)

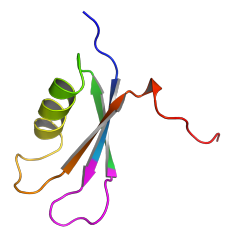

(c)
Figure 4. Grafting the FG motif onto three different scaffolds. This figure is showing three structures that successfully accepted the grafted the FG loop A: A domain of the human STAM1 VHS (PDB ID 3LDZ chain A) where the ATSEMNTAED sequence at positions 16-25 was replaced by the FG motif. B: An EAP domain protein from Staphylococcus aureus (PDB ID 1YN3 chain A) where the sequence ITVNGTSQNI at positions 164-173 was replaced by the FG sequence. C: The PAAB subunit of the PhenylacetateCoA Oxygenage from Ralstonia eutropha (PDB ID 3EGR chain B) where the VRSKQGLEHK sequence at positions 13-22 was replaced by the FG motif. $1 \mathrm{YN} 3$ was chosen since the native structure was easily forward folded using AbinitioRelax (figure 5). Thus it was easier to redesign this structure and it did result is a structure with a large energy gap between the desired structure and any other potential structure.

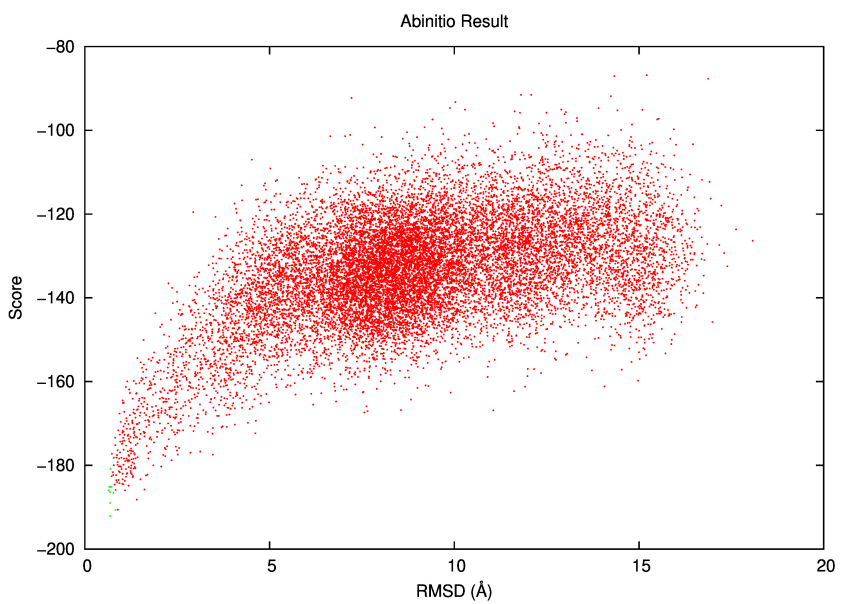

Figure 5. Folding simulation of the native 1YN3 protein structure. AbinitioRelax result of the native $1 \mathrm{YN} 3$ protein showing a successful simulation, a funnel shaped plot with the lowest simulated energy close to the predicted energy and RMSD of the structure.

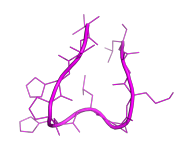

(a)

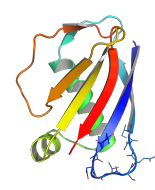

(b)

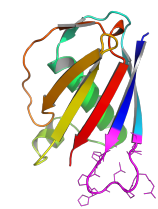

(c)
Figure 6. Stages of the grafting protocol. A: The native structure of the motif. B: The native structure of the Staphylococcus aureus EAP protein (PDB ID 1YN3) used here as a scaffold C: The final structure after the motif in purple was grafted onto the scaffold, then only the scaffold sequence designed (not the motif).

stability by maintaining an RMSD value between $2 \AA$ and $3 \AA$. The radius of gyration for all structures remained at around $13 \AA$ but with more variablity than the $300^{\circ} \mathrm{K}$ simulation.

These simulations can be compaired to the simulation of 

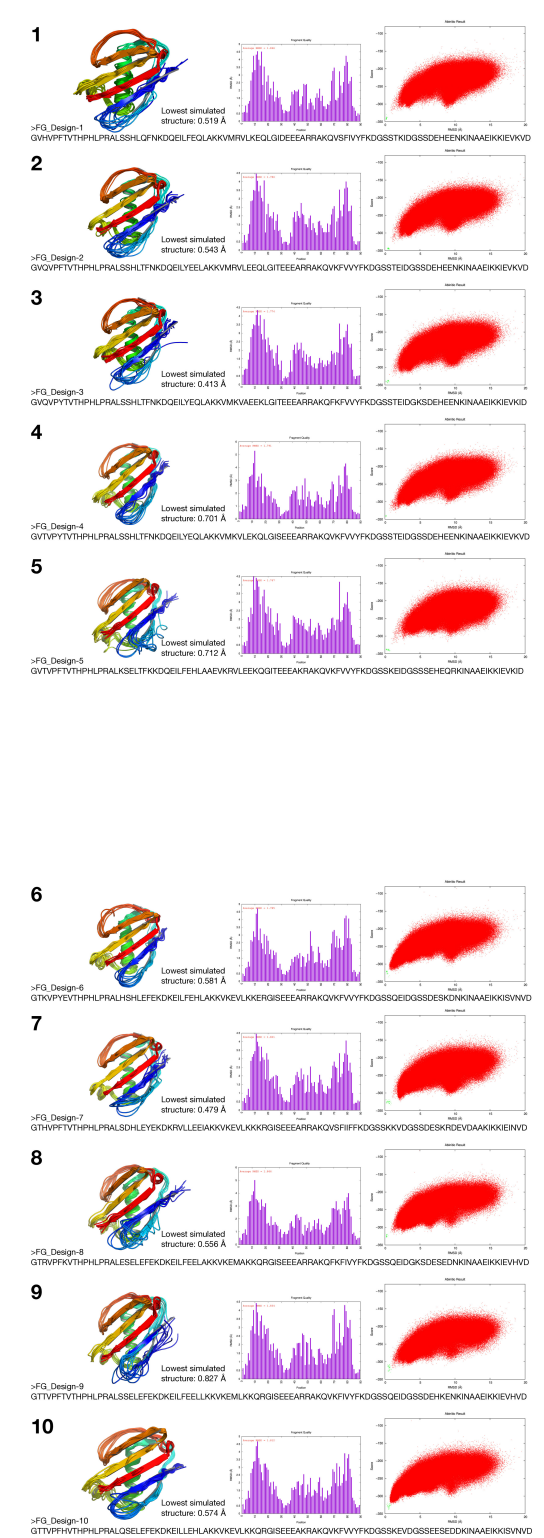

Figure 7. Final designed structures. Ten successfully designed structures that display the FG loop in its native three dimensional structure. The figure shows each designed structure (cartoon) superimposed onto the lowest energy and lowest RMSD structures from the AbinitioRelax simulation (wire) and the corresponding lowest RMSD value of the simulation, thus all structures were predicted to fold within a sub angstrom level of the designed structure giving high confidence that the proteins will have this fold when they are physically synthesised. Also showing are the FASTA sequences of each structure, the fragment quality used in each AbinitioRelax simulation, and the AbinitioRelax plot showing a successful funnel shaped plot for all structures. The green points in each folding simulation are the REF2015 (Rosetta Energy Function 2015) energy score values of the corresponding computationally designed structure after being relaxed thus indicating the lowest possible energy score for each structure and is thus used as a baseline to show were the global minima could be.

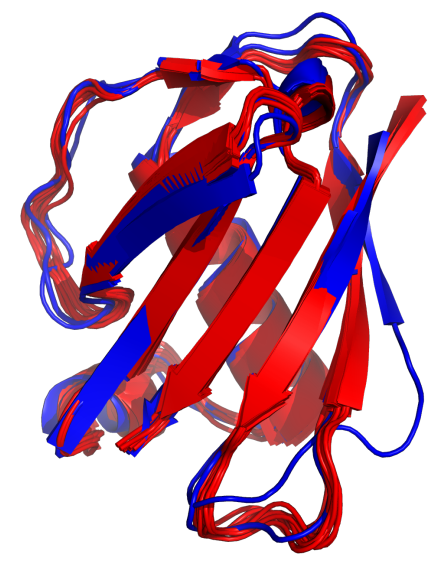

Figure 8. Swiss Model Predictions. The FASTA sequence of each of the designed proteins was used to predict their structure using Swiss Model [34] Here it can be seen that the predictions from the FASTA sequence predicts similar structures to the desined structures.

the original 1YN3 scaffold crystal structure from the Protein Databank (figure 11), where the structure was simulated at $300^{\circ} \mathrm{K}$ and $400^{\circ} \mathrm{K}$ using the same parameters. From the simulation at $300^{\circ} \mathrm{K}$ the structure showed a stable RMSD values (around $2 \AA$ ) with a value under $2 \AA$ at the end of the simulation, and a radius of gyration value around $13 \AA$. While the simulation at $400^{\circ} \mathrm{K}$ the structure showed a less stable structure with less stable RMSD values (above $2 \AA$ sometimes reaching $4 \AA$ ) with a value above $2 \AA$ at the end of the simulation, and a less stable radius of gyration value above $13 \AA$.

It can thus be argued that structures $3,4,5,6$, and 8 are the most stable structures at $300^{\circ} \mathrm{K}\left(26.85^{\circ} \mathrm{C}\right)$, while structure 5 is the most stable structures at $400^{\circ} \mathrm{K}\left(126.85^{\circ} \mathrm{C}\right)$.

\section{Conclusion}

This paper describes the protocol for computationally designing proteins that correctly display the three-dimensional structure of the FG strategic motif of the human IgE molecule. The motif was grafted onto the Staphylococcus aureus EAP protein (PDB ID 1YN3), which was used as a scaffold structure, then the scaffold/motif was sequence designed multiple times resulting in ten structures each with the same backbone, displaying the same motif in almost its native structure, yet each structure having a different sequence around the motif. Therefore, opening the possibility of using such protein structures as a vaccine and boosts against our own IgE to permanently shut down the allergy pathway regardless of the offending allergen (a pan-anti allergy vaccine). The resulting structures showed agreement in their final folds when simulated with the Rosetta AbinitioRelax folding algorithm, folding to sub angstrom levels when computationally folded from their amino acid sequence's primary structure. Nevertheless, the only definitive way to determine their realistic physical folds is to solve their structures through X-Ray crystallography or 


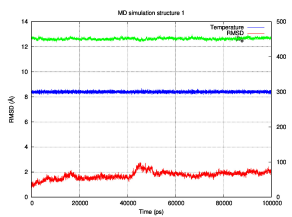

(a)

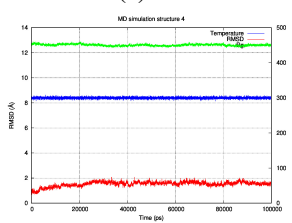

(d)

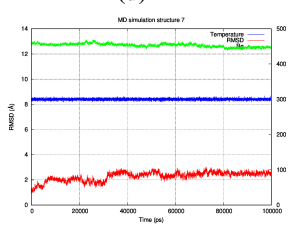

(g)

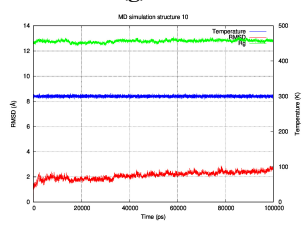

(j)

Figure 9. Molecular dynamics simulation at $300^{\circ} \mathrm{K}$. Molecular dynamics simulation of all the 10 designed structures at $300^{\circ} \mathrm{K}\left(26.85^{\circ} \mathrm{C}\right)$ for $100 \mathrm{~ns}$ (100,000 ps), all the structures showed a stable RMSD value (around $2 \AA$ ) where structures $3,4,6$, and 8 showed the highest stability while structures 7 , 9 , and 10 showed the lowest stability. The radius of gyration for all structures was also stable (around $13 \AA$.

NMR. Furthermore, the efficacy of the proteins in pushing the immune system into developing antibodies against our own IgE at a higher binding affinity than the IgE/FceRI receptor's binding affinity could not be computationally simulated, and thus must be tested on animals to reach a definitive answer. The script that was used to design these proteins is available at this GitHub repository, which includes an extensive README file and a video that explains how to use it.

This work performed initial testing of the hypothesis by employing in silico based methods for designing the proteins and did not include any experimental verifications. As a follow up, experimental verifications are required to further test this hypothesis which should include synthesis and purification of all the proteins in a bacterial host (the sequence of each protein is provided in figure 7), testing for binding between the synthesised proteins and known anti-IgE antibodies using the enzyme-linked immunosorbent assay (ELISA), modeling of all the structures through X-ray crystallography to ensure the FG loop is in the correct structure, and finally challanging animals for an immune reaction then testing their sera for binding to the proteins and the human IgE through ELISA, measuring the binding affinity of the antibodies to the proteins and human IgE through Surface Plasmon Resonance (SPR), and testing for $\mathrm{IgE} / \mathrm{Fc} \varepsilon \mathrm{RI}$ complex distruption through a cell-

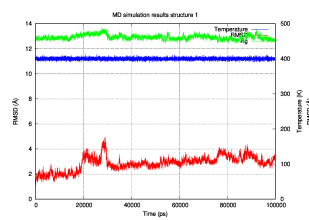

(a)

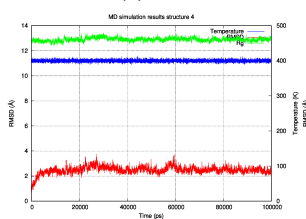

(d)

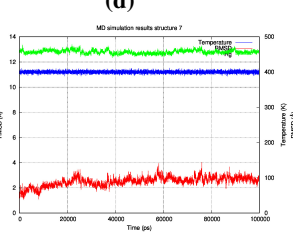

(g)

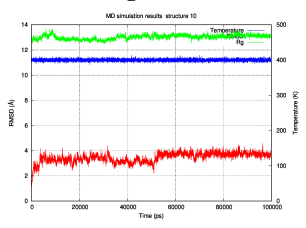

(j)

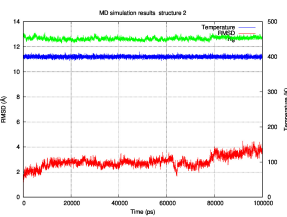

(b)

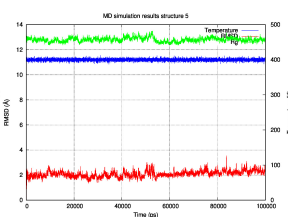

(e)

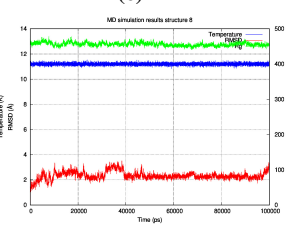

(h)

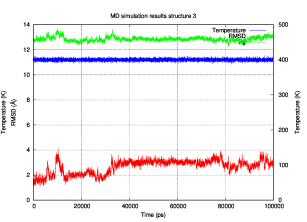

(c)

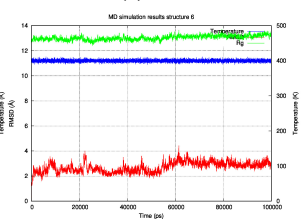

(f)

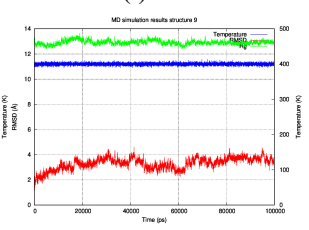

(i)
Figure 10. Molecular dynamics simulation at $400^{\circ} \mathrm{K}$. Molecular dynamics simulation of all the 10 designed structures at $400^{\circ} \mathrm{K}\left(126.85^{\circ} \mathrm{C}\right)$ for $100 \mathrm{~ns}$ $(100,000 \mathrm{ps})$, all the structures showed less RMSD stability (fluctuating up to $4 \AA$ and $5 \AA$ ). Structures $1,2,3,4,6,7,8,9$, and 10 showed low stability, reaching high RMSD values, while structures 5 showed the highest stability maintained at $2 \AA$ on average (occasionally reaching $3 \AA$ ). The radius of gyration for all structures remained at around $13 \AA$.

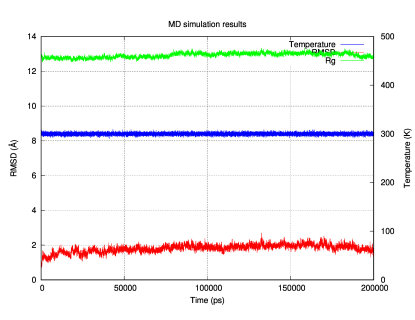

(a)

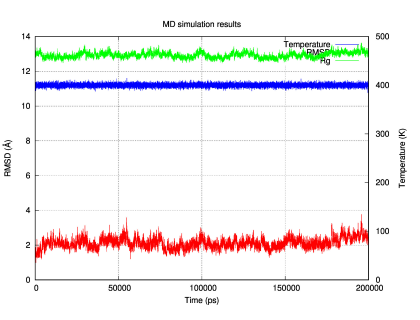

(b)
Figure 11. Molecular dynamics simulation of the original 1YN3 crystal structure structure at $300^{\circ} \mathrm{K}$ and $4^{\circ} 0^{\circ} \mathrm{K}$. Molecular dynamics simulation of the original $1 \mathrm{YN} 3$ structure from the Protein Databank at $300^{\circ} \mathrm{K}\left(26.85^{\circ} \mathrm{C}\right)$ and at $400^{\circ} \mathrm{K}\left(126.85^{\circ} \mathrm{C}\right)$ for $100 \mathrm{~ns}(100,000 \mathrm{ps})$. A: The $300^{\circ} \mathrm{K}$ simulation showed a structure stable at RMSD value around $2 \AA$ with a final RMSD value under $2 \AA$ at the end of the simulation, it also showed a stable radius of gyration value around $13 \AA$ at the end of the simulation. B: The $400^{\circ} \mathrm{K}$ simulation showed a less stable structure reaching an RMSD value of $3 \AA$ and sometimes coming close to $4 \AA$ with a final RMSD value above $2 \AA$ at the end of the simulation, it also showed a less stable radius of gyration value reaching above $13 \AA$ at the end of the simulation.

based mediator release assay [30]. 
bioRxiv preprint doi: https://doi.org/10.1101/2020.09.14.296392; this version posted March 11, 2021. The copyright holder for this preprint (which was not certified by peer review) is the author/funder. All rights reserved. No reuse allowed without permission.

Computationally Grafting an IgE Epitope onto a Scaffold: Implications for a Pan Anti-Allergy Vaccine Design — 8/9

\section{Availability of data and materials}

The code used in this project is available at this GitHub repository which includes an extensive README file and a video that explains how to use the script.

\section{Grant information}

The authors declared that no grants were involved in supporting this work.

\section{Competing interests}

The author has used these results to apply for a patent (filed by the institute) under application numberUS 16/988,076. The patent application is pending.

\section{Acknowledgements}

The corresponding author would like to thank the High Performance Computing Center at King Abdulaziz University for making available the Aziz high performance computer where the corresponding author was able to perform the Epitope Grafting search, the AbinitioRelax folding simulations, and the molecular dynamics simulations.

\section{References}

[1] Andrew Leaver-Fay, Brian Kuhlman, and Jack Snoeyink. Rotamer-pair energy calculations using a trie data structure. In Rita Casadio and Gene Myers, editors, Algorithms in Bioinformatics, pages 389-400, Berlin, Heidelberg, 2005. Springer Berlin Heidelberg.

[2] Mihai L. Azoitei, Yih-En Andrew Ban, Jean-Philippe Julien, Steve Bryson, Alexandria Schroeter, Oleksandr Kalyuzhniy, Justin R. Porter, Yumiko Adachi, David Baker, Emil F. Pai, and William R. Schief. Computational design of high-affinity epitope scaffolds by backbone grafting of a linear epitope. Journal of Molecular Biology, 415(1):175 - 192, 2012.

[3] Mihai L. Azoitei, Bruno E. Correia, Yih-En Andrew Ban, Chris Carrico, Oleksandr Kalyuzhniy, Lei Chen, Alexandria Schroeter, Po-Ssu Huang, Jason S. McLellan, Peter D. Kwong, David Baker, Roland K. Strong, and William R. Schief. Computation-guided backbone grafting of a discontinuous motif onto a protein scaffold. Science, 334(6054):373376, 2011.

[4] Richard Bonneau, Charlie E.M Strauss, Carol A Rohl, Dylan Chivian, Phillip Bradley, Lars Malmström, Tim Robertson, and David Baker. De novo prediction of three-dimensional structures for major protein families. Journal of Molecular Biology, 322(1):65 - 78, 2002.

[5] Richard Bonneau, Jerry Tsai, Ingo Ruczinski, Dylan Chivian, Carol Rohl, Charlie E. M. Strauss, and David Baker. Rosetta in casp4: Progress in ab initio protein structure prediction. Proteins: Structure, Function, and Bioinformatics, 45(S5):119-126, 2001.

[6] Philip Bradley, Kira M. S. Misura, and David Baker. Toward highresolution de novo structure prediction for small proteins. Science, 309(5742):1868-1871, 2005.

[7] C. Von Pirquet. MÜnchener medizinische wochenschrift. Allergie, 1909.

[8] Gautam Dantas, Brian Kuhlman, David Callender, Michelle Wong, and David Baker. A large scale test of computational protein design: Folding and stability of nine completely redesigned globular proteins. Journal of Molecular Biology, 332(2):449 - 460, 2003.
[9] Brian V Geisbrecht, Brent Y Hamaoka, Benjamin Perman, Adam Zemla, and Daniel J Leahy. The crystal structures of eap domains from staphylococcus aureus reveal an unexpected homology to bacterial superantigens. Journal of Biological Chemistry, 280(17):17243-17250, 2005.

[10] G. B. Gidaro, F. Marcucci, L. Sensi, C. Incorvaia, F. Frati, and G. Ciprandi. The safety of sublingual-swallow immunotherapy: an analysis of published studies. Clinical \& Experimental Allergy, 35(5):565$571,2005$.

[11] Xiaozhen Hu, Huanchen Wang, Hengming Ke, and Brian Kuhlman. High-resolution design of a protein loop. Proceedings of the National Academy of Sciences, 104(45):17668-17673, 2007.

[12] Kimishige Ishizaka, Teruko Ishizaka, and Margaret M. Hornbrook. Physico-chemical properties of human reaginic antibody. The Journal of Immunology, 97(1):75-85, 1966.

[13] Brian Kuhlman, Gautam Dantas, Gregory C. Ireton, Gabriele Varani, Barry L. Stoddard, and David Baker. Design of a novel globular protein fold with atomic-level accuracy. Science, 302(5649):1364-1368, 2003.

[14] L. Noon. Prophylactic inoculation against hay fever. Lancet, 177:15721573, 1911.

[15] A. Leaver-fay, B. Kuhlman, J. Snoeyink, Andrew Leaver-fay, Brian Kuhlman, and Jack Snoeyink. An adaptive dynamic programming algorithm for the side chain placement problem. In In Pacific Symposium on Biocomputing, pages 17-28. World Scientific, 2005.

[16] Andrew Leaver-Fay, Michael Tyka, Steven M. Lewis, Oliver F. Lange, James Thompson, Ron Jacak, Kristian W. Kaufman, P. Douglas Renfrew, Colin A. Smith, Will Sheffler, Ian W. Davis, Seth Cooper, Adrien Treuille, Daniel J. Mandell, Florian Richter, Yih-En Andrew Ban, Sarel J. Fleishman, Jacob E. Corn, David E. Kim, Sergey Lyskov, Monica Berrondo, Stuart Mentzer, Zoran Popović, James J. Havranek, John Karanicolas, Rhiju Das, Jens Meiler, Tanja Kortemme, Jeffrey J. Gray, Brian Kuhlman, David Baker, and Philip Bradley. Chapter nineteen rosetta3: An object-oriented software suite for the simulation and design of macromolecules. In Michael L. Johnson and Ludwig Brand, editors, Computer Methods, Part C, volume 487 of Methods in Enzymology, pages 545 - 574. Academic Press, 2011.

[17] David B Lewis. Allergy immunotherapy and inhibition of th2 immune responses: a sufficient strategy? Current Opinion in Immunology, 14(5):644-651, 2002.

[18] Martin D Chapman. Allergens. Elsevier, 1998.

[19] R. Movérare, L. Elfman, E. Vesterinen, T. Metso, and T. Haahtela. Development of new ige specificities to allergenic components in birch pollen extract during specific immunotherapy studied with immunoblotting and pharmacia cap system ${ }^{\mathrm{TM}}$. Allergy, 57(5):423-430, 2002.

[20] H. Okada, C. Kuhn, H. Feillet, and J.-F. Bach. The 'hygiene hypothesis' for autoimmune and allergic diseases: an update. Clinical \& Experimental Immunology, 160(1):1-9, 2010.

[21] Ruby Pawankar, GW Canonica, ST Holgate, RF Lockey, and M Blaiss. World allergy organization (wao) white book on allergy. Wisconsin: World Allergy Organisation, 2011.

[22] Leonard G Presta, SJ Lahr, RL Shields, JP Porter, CM Gorman, BM Fendly, and PM Jardieu. Humanization of an antibody directed against ige. The Journal of Immunology, 151(5):2623-2632, 1993.

[23] Srivatsan Raman, Robert Vernon, James Thompson, Michael Tyka, Ruslan Sadreyev, Jimin Pei, David Kim, Elizabeth Kellogg, Frank DiMaio, Oliver Lange, et al. Structure prediction for casp8 with all-atom refinement using rosetta. Proteins: Structure, Function, and Bioinformatics, 77(S9):89-99, 2009. 
bioRxiv preprint doi: https://doi.org/10.1101/2020.09.14.296392; this version posted March 11, 2021. The copyright holder for this preprint (which was not certified by peer review) is the author/funder. All rights reserved. No reuse allowed without permission.

Computationally Grafting an IgE Epitope onto a Scaffold: Implications for a Pan Anti-Allergy Vaccine Design — 9/9

[24] Kim T Simons, Charles Kooperberg, Enoch Huang, and David Baker. Assembly of protein tertiary structures from fragments with similar local sequences using simulated annealing and bayesian scoring functions. Journal of molecular biology, 268(1):209-225, 1997.

[25] Kim T Simons, Ingo Ruczinski, Charles Kooperberg, Brian A Fox, Chris Bystroff, and David Baker. Improved recognition of native-like protein structures using a combination of sequence-dependent and sequenceindependent features of proteins. Proteins: Structure, Function, and Bioinformatics, 34(1):82-95, 1999.

[26] David P Strachan. Hay fever, hygiene, and household size. BMJ: British Medical Journal, 299(6710):1259, 1989.

[27] David P Strachan. Family size, infection and atopy: the first decade of the'hygiene hypothesis'. Thorax, 55(Suppl 1):S2, 2000.

[28] Leena von Hertzen, T Klaukka, H Mattila, and T Haahtela. Mycobacterium tuberculosis infection and the subsequent development of asthma and allergic conditions. Journal of allergy and clinical immunology, 104(6):1211-1214, 1999.

[29] LLC Schrödinger. The pymol molecular graphics system, version 1.8. Schrödinger, LLC New York, 2015.

[30] Sari Sabban. Development of an in vitro model system for studying the interaction of Equus caballus IgE with its high-affinity FcERI receptor. $\mathrm{PhD}$ thesis, University of Sheffield, 2011.

[31] Alford, Rebecca F. and Leaver-Fay, Andrew and Jeliazkov, Jeliazko R. and O'Meara, Matthew J. and DiMaio, Frank P. and Park, Hahnbeom and Shapovalov, Maxim V. and Renfrew, P. Douglas and Mulligan, Vikram K. and Kappel, Kalli and Labonte, Jason W. and Pacella, Michael S. and Bonneau, Richard and Bradley, Philip and Dunbrack, Roland L. and Das, Rhiju and Baker, David and Kuhlman, Brian and Kortemme, Tanja and Gray, Jeffrey J. The Rosetta All-Atom Energy Function for Macromolecular Modeling and Design Journal of Chemical Theory and Computation, 13(6):3031-3048, 2017

[32] Peter J A Cock, Tiago Antao, Jeffrey T Chang, Brad A Chapman, Cymon J Cox, Andrew Dalke, Iddo Friedberg, Thomas Hamelryck, Frank Kauff, Bartek Wilczynski, Michiel J L de Hoon Biopython: freely available python tools for computational molecular biology and bioinformatics Bioinformatics, 25(11):1422-1423, 2009

[33] Koga, Nobuyasu and Tatsumi-Koga, Rie and Liu, Gaohua and Xiao, Rong and Acton, Thomas B and Montelione, Gaetano T and Baker, David Principles for designing ideal protein structures Nature, 491(7423):222-227, 2012

[34] Buchan, Daniel and Jones, David The PSIPRED Protein Analysis Workbench: 20 years on Nucleic acids research, 7(47):W402-W407, 2019

[35] Waterhouse, Andrew and Bertoni, Martino and Bienert, Stefan and Studer, Gabriel and Tauriello, Gerardo and Gumienny, Rafal and Heer, Florian T and de Beer, Tjaart A P and Rempfer, Christine and Bordoli, Lorenza and others SWISS-MODEL: homology modelling of protein structures and complexes Nucleic acids research, 46(W1):W296-W303, 2018

[36] Bekker, $\mathrm{H}$ and Berendsen, HJC and Dijkstra, EJ and Achterop, S and Van Drunen, R and Van der Spoel, D and Sijbers, A and Keegstra, H and Reitsma, B and Renardus, MKR Gromacs: A parallel computer for molecular dynamics simulations Physics computing, 92:252-256, 1993

[37] Berendsen, Herman JC and van der Spoel, David and van Drunen, Rudi GROMACS: a message-passing parallel molecular dynamics implementation Computer physics communications, 91(1-3):43-56, 1995
[38] Lindahl, Erik and Hess, Berk and Van Der Spoel, David GROMACS 3.0: a package for molecular simulation and trajectory analysis Molecular modeling annual, 7(8):306-317, 2001

[39] Van Der Spoel, David and Lindahl, Erik and Hess, Berk and Groenhof, Gerrit and Mark, Alan E and Berendsen, Herman JC GROMACS: fast, flexible, and free Journal of computational chemistry, 26(16):1701-1718, 2005

[40] Hess, Berk and Kutzner, Carsten and Van Der Spoel, David and Lindahl, Erik GROMACS 4: algorithms for highly efficient, load-balanced, and scalable molecular simulation Journal of chemical theory and computation, 4(3):435-447, 2008

[41] Pronk, Sander and Páll, Szilárd and Schulz, Roland and Larsson, Per and Bjelkmar, Pär and Apostolov, Rossen and Shirts, Michael R and Smith, Jeremy $\mathrm{C}$ and Kasson, Peter M and van der Spoel, David and others GROMACS 4.5: a high-throughput and highly parallel open source molecular simulation toolkit Bioinformatics, 29(7):845-854, 2013

[42] Páll, Szilárd and Abraham, Mark James and Kutzner, Carsten and Hess, Berk and Lindahl, Erik Tackling exascale software challenges in molecular dynamics simulations with GROMACS International conference on exascale applications and software, 3-27, 2014

[43] Abraham, Mark James and Murtola, Teemu and Schulz, Roland and Páll, Szilárd and Smith, Jeremy C and Hess, Berk and Lindahl, Erik GROMACS: High performance molecular simulations through multilevel parallelism from laptops to supercomputers SoftwareX, 1:19-25, 2015 